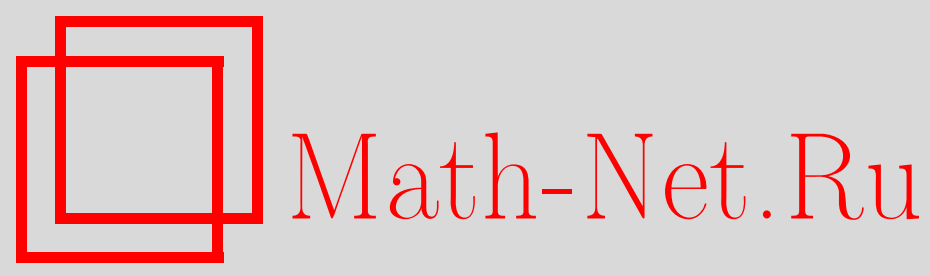

S. Yu. Katyshev, A. V. Zyazin, A. V. Baryshnikov, Использование неассоциативных структур при построении гомоморфных криптографических механизмов, Матем. вопр. криптогр., 2020, том 11, выпуск 3, 31-39

DOI: https://doi.org/10.4213/mvk330

Использование Общероссийского математического портала Math-Net.Ru подразумевает, что вы прочитали и согласны с пользовательским соглашением http://www.mathnet.ru/rus/agreement

Параметры загрузки:

IP: 18.234 .156 .22

26 апреля 2023 г., 17:06:52

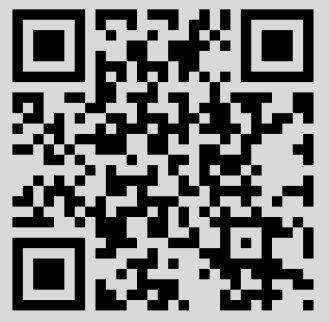




\title{
Application of non-associative structures for construction of homomorphic cryptosystems*
}

\author{
S. Yu. Katyshev ${ }^{1}$, A. V. Zyazin ${ }^{1}$, A. V. Baryshnikov ${ }^{2}$ \\ ${ }^{1}$ Russian Technological University (MIREA), Moscow \\ ${ }^{2}$ Certification Research Center LLC, Moscow
}

Получено 06.II.2019

\begin{abstract}
Homomorphic encoding allows to perform certain mathematical operations with the encoded text and to get the encoded outcome that corresponds to the result of operations processed with a plaintext. There exist both fully homomorphic and partially homomorphic options (with respect to one or more operations). For practical use of such an encoding it is necessary to have a homomorphism with respect for at least one operation. Using non-associative operations, we construct in this paper an example of a cryptosystem based on the El-Gamal system that is homomorphic with respect to two on-going operations: a group and a quasigroup ones.
\end{abstract}

Keywords: public-key cryptosystem, homomorphic encryption, non-associative algebraic structures

Использование неассоциативных структур при построении гомоморфных криптографических механизмов

\section{С. Ю. Катышев ${ }^{1}$, А. В. Зязин ${ }^{1}$, А. В. Барышников ${ }^{2}$}

${ }^{1}$ Российский технологический университет (МИРЭА), Москва

${ }^{2}$ ООО «Центр сертификационных исследований», Москва

Аннотация. Гомоморфное шифрование позволяет выполнять некоторые математические преобразования с шифрованным текстом, в результате которых получается шифрованный текст, соответствующий результату выполнения заданного преобразования открытого текста. Известны так называемые полностью гомоморфные и частично гомоморфные шифрсистемы, различающиеся количеством допустимых для выполнения преобразований. Для построения подобных шифрсистем необходимо иметь гомоморфизм относительно хотя бы одной из выполняемых операций. В работе с использованием

* The article was submitted by the Organizing Committee of the Symposium CTCrypt'2019. 
неассоциативных операций и системы Эль-Гамаля построен пример шифрсистемы, гомоморфной относительно двух выполняемых операций: групповой и квазигрупповой.

Ключевые слова: асимметричная криптография, гомоморфное шифрование, неассоциативные алгебраические структуры

\section{Introduction}

Fully Homomorphic Encryption (FHE) is a specific cryptographic primitive that allows to compute the ciphertexts $\operatorname{Enc}\left(f\left(m_{1}, \ldots, m_{t}\right)\right)$ as a function of the encrypted data $\operatorname{Enc}\left(m_{1}\right), \operatorname{Enc}\left(m_{2}\right), \ldots, \operatorname{Enc}\left(m_{t}\right)$ and of the arbitrary function $f$ without knowing the decryption key and the original input data $m_{1}, \ldots, m_{t}$. If the encrypted data $\operatorname{Enc}\left(m_{1}\right), \operatorname{Enc}\left(m_{2}\right), \ldots, \operatorname{Enc}\left(m_{t}\right)$ allows to compute the ciphertext $\operatorname{Enc}\left(f\left(m_{1}, \ldots, m_{t}\right)\right)$ only for some functions $f$, then the primitive is called a partially homomorphic encryption.

Cryptographically strong FHE systems may provide new opportunities to maintain information security in such areas like cloud computing, medical and financial data processing because they allow to operate data without its preliminary deciphering in an untrusted environment. The very idea of the FHE was firstly proposed in 1978 in [12] but found no practical implementation.

The first successful attempt to create a FHE system was the system proposed by Goldwasser-Micali in 1982 [7]. However, this cryptosystem was homomorphic only for one operation: modulo 2 addition.

Furthermore, there exist a number of systems presented that were homomorphic only in one operation, such as widely known El-Gamal and RSA cryptosystems. The question on existence of FHE remained unsolved. The first homomorphism was developed by D. Boneh, E.-J. Goh and K. Nissim in the system elaborated in 2005 [5] allowing the computation of any number of additions and one multiplication of the encrypted messages.

An encryption system homomorphic with respect to addition and multiplication in the residue ring was firstly proposed by Gentry [6] in 2009. Afterwards, there appeared more than a dozen papers providing alternative options for encrypting systems to be fully homomorphic over residue rings. A detailed overview of these papers may be found in [2].

Despite the use of a certain number of cryptosystems proposed in realworld system, it was already proved that the FHE systems over the residue rings are vulnerable to the known-plaintext attacks [2]. In this regard, a particular interest may be payed to the homomorphic systems based on non- 
associative structures. Such systems shouldn't be obligatory homomorphic over the residue rings but they still allow to solve a number of practical issues.

It was already proposed in [8] to use some classes of groupoids for encryption systems which are homomorphic with respect to a unique operation. In this paper we consider the extension of the El-Gamal [9] cryptosystem to quasi-groups linear over the Abelian group. The resulting system possesses the homomorphic property with respect to two operations at once: group and quasi-group.

\section{Basic definitions}

Let Enc, Dec be an encrypting and decrypting functions of a cryptosystem $A$. Such a cryptosystem is called homomorphic with respect to an n-ary operation $*\left(a_{1}, \ldots, a_{n}\right)[13]$ if there exists an effective algorithm $M$ which transforms (for any set of plaintexts $\left.\left(m_{1}, \ldots, m_{n}\right)\right)$ the input set $\left(\operatorname{Enc}\left(m_{1}\right)\right.$, $\left.\ldots, \operatorname{Enc}\left(m_{n}\right)\right)$ into output $C$ such that

$$
\operatorname{Dec}(C)=*\left(m_{1}, \ldots, m_{n}\right) .
$$

A particular case of such encryption systems are systems for which

$$
\operatorname{Dec}\left(*\left(\operatorname{Enc}\left(m_{1}\right), \ldots, \operatorname{Enc}\left(m_{n}\right)\right)\right)=*\left(m_{1}, \ldots, m_{n}\right) .
$$

In other words, homomorphic encryption allows to produce certain mathematical operations with encrypted texts and obtain an encrypted result that corresponds to the results performed with plaintexts.

Following [10], for an element $g$ of a groupoid $(\Omega, *)$ and given $r, l \in \mathbb{N}$, we define the right $r$-th and left $l$-th powers respectively by the equalities:

$$
g^{[r]}=\underbrace{(\ldots((g * g) * g) \ldots)}_{r \text { factors }}, \quad{ }^{[l]} g=\underbrace{(\ldots(g *(g * g)) \ldots)}_{\text {l factors }} .
$$

We say that $g$ has commuting right powers, or that $g$ is a CRP-element, if

$$
\forall m, n \in \mathbb{N}: \quad g^{[m][n]}=g^{[n][m]} .
$$

If this identity is valid for any element $g \in \Omega$, then we say that $(\Omega, *)$ is a CRP-groupoid.

Similarly, using the identity

$$
\forall m, n \in \mathbb{N}: \quad{ }^{[m][n]} g={ }^{[n][m]} g,
$$


we define elements and groupoids with commuting left powers, CLP-elements and CLP-groupoids, respectively.

Let us say that a groupoid $(\Omega, *)$ is a groupoid with commuting powers (CP-groupoid) if it is a CLP- and CRP-groupoid and moreover, for any $g \in \Omega$ and any $l, r \in \mathbb{N}$ the following equality holds:

$$
{ }^{[l]}\left(g^{[r]}\right)=\left({ }^{[l]} g\right)^{[r]} .
$$

Example. Let $(\Omega,+)$ be an Abelian group. Fixing two commuting automorphisms $\sigma, \tau \in \operatorname{Aut}(\Omega)(\sigma \tau=\tau \sigma)$ we define a new operation $*$ on $\Omega$ by the following condition:

$$
\forall x, y \in \Omega \quad x * y=\sigma(x)+\tau(y) .
$$

So we obtain a groupoid $(\Omega, *)$, which is a quasi-group. Such quasi-groups form a class of medial quasi-groups i.e. quasi-groups with the identity $(x * y) *(u * v)=(x * u) *(y * v)$ [1]. According to [10] quasi-group $(\Omega, *)$ is a CP-groupoid.

Some medial quasi-groups have already been used in [8] to create encryption system for homomorphic quasi-group operations $*$.

\section{Cryptosystem description}

Let $(\Omega,+)$ be an Abelian group, and $(\Omega, *)$ be a medial quasi-group with operation (2). The commuting powers allow to extend El-Gamal cryptosystems on quasi-group $(\Omega, *)$.

\section{Cryptosystem 1.}

1. Public key generation. Selecting an element $g \in \Omega$, user $A$ generates an arbitrary (secret) number $r_{A}$ and computes $g_{A}=g^{\left[r_{A}\right]}$. The pair $\left(g, g_{A}\right)$ is the public key of user $A$.

2. Encryption. In order to encrypt a message $m \in \Omega$ user $B$ generates an arbitrary (secret) number $r_{B}$ and computes $g_{B}=g^{\left[r_{B}\right]}$. Then he computes $g_{A}^{\left[r_{B}\right]}$ and $m_{A B}=m+g_{A}^{\left[r_{B}\right]}$. His ciphertext is the pair $\left(g_{B}, m_{A B}\right)$.

3. Decrypting. In order to decrypt a ciphertext $\left(g_{B}, m_{A B}\right)$ user $A$ computes $g_{B}^{\left[r_{A}\right]}$ and finds $x=m_{A B}-g_{B}^{\left[r_{A}\right]}$.

Denote by $\operatorname{AUT}(\sigma, t)$ the complexity of calculation of $\sigma^{t}(a)$ for an arbitrary element $a$ of the semigroup $(\Omega,+)$.

Theorem 1. The Cryptosystem 1 operates correctly, the complexity of a message encryption is estimated by $O\left(\operatorname{AUT}(\sigma,|\Omega|) \log _{2}(|\Omega|)\right)$ operations in the group $(\Omega,+)$. 
Proof. Let $\left(g, g_{A}\right)$ be a public key for user $A$, and $\left(g_{B}, m_{A B}\right)$ be the result of encoding of an arbitrary message $m \in \Omega$ with this key. To decrypt the message user $A$ computes $x=m_{A B}-g_{B}^{\left[r_{A}\right]}=m+g_{A}^{\left[r_{B}\right]}-g_{B}^{\left[r_{A}\right]}$. Due to commutation of the right powers in the quasi-group $(\Omega, *)$, we have

$$
g_{A}^{\left[r_{B}\right]}=g^{\left[r_{A}\right]\left[r_{B}\right]}=g_{B}^{\left[r_{A}\right]} .
$$

Therefore, $x=m+g_{A}^{\left[r_{B}\right]}-g_{B}^{\left[r_{A}\right]}=m$, and the decoding is accomplished correctly.

The estimation in Theorem 1 follows from the fact that complexity of computation of the element $g^{[k]}$ in the quasi-group $(\Omega, *)$, according to [10], is estimated by $O\left(\operatorname{AUT}(\sigma, k) \log _{2}(k)\right)$ operations in the group $(\Omega,+)$.

Remark 1. Conventionally, automorphisms are defined by the action on generators of the group. In this case, according to [10], the complexity of exponentiation, and hence the encryption in the Cryptosystem 1 is estimated as $O(\log |\Omega|)$. If automorphisms $\sigma, \tau$ have small order in comparison with the order of the group, this valuation equals the estimation of the complexity due to the classical El-Gamal scheme.

Note that the complexity of revelation of the secret key by an observer having access to the open information $g, g^{\left[r_{A}\right]}, g^{\left[r_{B}\right]}$ does not exceed the complexity of the right discrete logarithm in the groupoid, i.e. the complexity of solving the equation

$$
g^{[x]}=h .
$$

Remark 2. The complexity of the right discrete logarithm in the groupoid depends both on the groupoid structure and on its representation. The solution to the problem within different structures was investigated in $[3,4,10,11]$.

A natural generalization of Cryptosystem 1 consists in combination of right and left powers.

\section{Cryptosystem 2.}

1. Public key generation. User $A$ chooses an element $g \in \Omega$, a natural numbers $r \leqslant n$, an ordered set of numbers $a_{1}, \ldots, a_{n} \in \mathbb{N}$, and computes

$$
g_{A}={ }^{\left[a_{1}\right] \ldots\left[a_{r}\right]} g^{\left[a_{r+1}\right] \ldots\left[a_{n}\right]} .
$$

User's $A$ public key is the pair $\left(g, g_{A}\right)$. 
2. Encryption. In order to encrypt a message $m \in \Omega$, user $B$ generates natural numbers $t \leqslant k$, an ordered set of numbers $b_{1}, \ldots, b_{k} \in \mathbb{N}$, and computes

$$
g_{B}=\left[b_{1}\right] \ldots\left[b_{t}\right] g^{\left[b_{t+1}\right] \ldots\left[b_{k}\right]} .
$$

Then he computes $g_{A B}=\left[b_{1}\right] \ldots\left[b_{t}\right] g_{A}^{\left[b_{t+1}\right] \ldots\left[b_{k}\right]}$ and $m_{A B}=m+g_{A B}$. The ciphertext is the pair $\left(g_{B}, m_{A B}\right)$.

3. Decrypting. In order to decrypt a ciphertext $\left(g_{B}, m_{A B}\right)$, user $A$ computes $g_{B A}=\left[a_{1}\right] \ldots\left[a_{r}\right] g_{B}^{\left[a_{r+1}\right] \ldots\left[a_{n}\right]}$ and finds $x=m_{A B}-g_{B A}$.

Theorem 2. The Cryptosystem 2 operates correctly. The complexity of message encryption is estimated by $O\left(N \cdot \operatorname{AUT}(\sigma,|\Omega|) \log _{2}(|\Omega|)\right)$ operations in the group $(\Omega,+)$, where $N=\max (n, k)$.

Proof. The same as for the Theorem 1.

Remark 3. It is natural to choose $n$ and $k$ not exceeding the logarithm of the size of the set $\Omega$.

When using the Cryptosystem 2, the recovery of a message by the user from the ciphertext is not harder than the problem of generalized discrete logarithm [10], i.e. of finding some pair of positive integers $u, v$ and some set $\left(x_{1}, \ldots, x_{v}\right) x_{i} \in \mathbb{N}, i \in \overline{1, v}$, satisfying the equation

$$
\left[x_{1}\right] \ldots\left[x_{u}\right] g^{\left[x_{u+1}\right] \ldots\left[x_{v}\right]}=h,
$$

if such numbers exist. At the moment, common approaches to a solution of the problem are not described yet. Some approaches are developed in [4].

It is well-known that El-Gamal scheme is homomorphic in regard to group operations. Systems constructed by means of medial quasi-groups also are homomorphic in regard to group operations. For further presentation we need an additional Lemma.

Lemma 1. For any elements $a$ and $b$ of the medial quasi-group $(\Omega, *)$, and for any natural numbers $n$ and $k$ the following equations are true:

1) $(a+b)^{[n]}=a^{[n]}+b^{[n]}$,

2) ${ }^{[k]}(a+b)={ }^{[k]} a+{ }^{[k]} b$,

3) ${ }^{[k]}(a+b)^{[n]}={ }^{[k]} a^{[n]}+{ }^{[k]} b^{[n]}$.

Proof. According to [10], for any element $g$ of the quasi-group $(\Omega, *)$ the following equation is satisfied:

$$
g^{[n]}=\sigma^{n-1}(g)+\sigma^{n-2}(\tau(g))+\ldots+\sigma(\tau(g))+\tau(g) .
$$


If we take $g=a+b$, we get

$$
(a+b)^{[n]}=\sigma^{n-1}(a+b)+\sigma^{n-2}(\tau(a+b))+\ldots+\sigma(\tau(a+b))+\tau(a+b) .
$$

As $\sigma$ and $\tau$ are homomorphisms of the group $(\Omega,+)$, for any natural number $k$ we have

$$
\sigma^{k}(\tau(a+b))=\sigma^{k}(\tau(a)+\tau(b))=\sigma^{k}(\tau(a))+\sigma^{k}(\tau(b)) .
$$

Using this equality we get

$$
\begin{aligned}
&(a+b)^{[n]}=\left(\sigma^{n-1}(a)+\sigma^{n-2}(\right.(a)) \\
&+\ldots+\tau(a)) \\
&+\left(\sigma^{n-1}(b)+\sigma^{n-2}(\tau(b))+\ldots+\tau(b)\right) .
\end{aligned}
$$

The proof of the first equality is finished with the implementation of the identity (5) for elements $a$ and $b$.

The second equality is proved in the same way, using the identity described in [10]:

$$
{ }^{[k]} g=\tau^{k-1}(g)+\tau^{k-2}(\sigma(g))+\ldots+\tau(\sigma(g))+\sigma(g) .
$$

The last equality follows from the first two ones.

Theorem 3. The cryptosystems 1 and 2 are homomorphic with respect to the group operation + .

Proof. Since Cryptosystem 1 is a particular case of the system 2, we need to consider only the Cryptosystem 2.

Let $\operatorname{Enc}(m)$, Dec $(c)$ be the algorithms of the encryption and decryption, defined in the stages 2 and 3 of the Cryptosystem 2. Let us check that they are homomorphic with respect to the operation + .

Let $c_{1}=\left(g_{1}, s_{1}\right), c_{2}=\left(g_{2}, s_{2}\right)$ be ciphertexts obtained as a result of encryption of the messages $m_{1}$ and $m_{2}$ under a public key $k=\left(g, g_{A}\right)$.

Apply the algorithm of the decryption Dec to $c_{1}+c_{2}=\left(g_{1}+g_{2}, s_{1}+s_{2}\right)$ :

$$
\operatorname{Dec}\left(c_{1}+c_{2}\right)=\left(s_{1}+s_{2}\right)-{ }^{\left[a_{1}\right] \ldots\left[a_{r}\right]}\left(g_{1}+g_{2}\right)^{\left[a_{r+1}\right] \ldots\left[a_{n}\right]} .
$$

Using Lemma 1 several times, we get

$$
{ }^{\left[a_{1}\right] \ldots\left[a_{r}\right]}\left(g_{1}+g_{2}\right)^{\left[a_{r+1}\right] \ldots\left[a_{n}\right]}={ }^{\left[a_{1}\right] \ldots\left[a_{r}\right]} g_{1}^{\left[a_{r+1}\right] \ldots\left[a_{n}\right]}+{ }^{\left[a_{1}\right] \ldots\left[a_{r}\right]} g_{2}^{\left[a_{r+1}\right] \ldots\left[a_{n}\right]} .
$$

According to the definition of Dec

$$
s_{1}-{ }^{\left[a_{1}\right] \ldots\left[a_{r}\right]} g_{1}^{\left[a_{r+1}\right] \ldots\left[a_{n}\right]}=\operatorname{Dec}\left(c_{1}\right), s_{2}-{ }^{\left[a_{1}\right] \ldots\left[a_{r}\right]} g_{2}^{\left[a_{r+1}\right] \ldots\left[a_{n}\right]}=\operatorname{Dec}\left(c_{2}\right) .
$$


We obtain

$$
\operatorname{Dec}\left(c_{1}+c_{2}\right)=\operatorname{Dec}\left(c_{1}\right)+\operatorname{Dec}\left(c_{2}\right) \text {. }
$$

Let us turn to consideration of the quasi-group operation $*$.

Lemma 2. For any elements $a, b, c, d \in \Omega$ and natural numbers $n$ and $k$, the following equalities hold:

1) ${ }^{[k]}(a * b)^{[n]}={ }^{[k]} a^{[n]} *{ }^{[k]} b^{[n]}$,

2) $(a+b) *(c+d)=a * c+b * d$.

Proof. According to the definition of the operation $*$ we have $a * b=$ $\sigma(a)+\tau(b)$. Therefore,

$\sigma(a * b)=\sigma(\sigma(a)+\tau(b))=\sigma^{2}(a)+\sigma(\tau(b))=\sigma^{2}(a)+\tau(\sigma(b))=\sigma(a) * \sigma(b)$.

In particular, $\sigma\left(a^{[k]}\right)=(\sigma(a))^{[k]}$ and, similarly, $\tau\left(a^{[k]}\right)=(\tau(a))^{[k]}$. To obtain the first equality we need to apply Lemma 1 to the elements $\sigma(a)$ and $\tau(b)$.

Let us consider the second equality.

$$
(a+b) *(c+d)=\sigma(a+b)+\tau(c+d) .
$$

As $\sigma$ and $\tau$ are homomorphisms of the group $(\Omega,+)$,

$$
\sigma(a+b)=\sigma(a)+\sigma(b), \quad \tau(c+d)=\tau(c)+\tau(d) .
$$

If we rearrange the summands, we get

$$
(a+b) *(c+d)=(\sigma(a)+\tau(c))+(\sigma(b)+\tau(d)) .
$$

Using the definition of the operation $*$ once again, we get the required equality.

Theorem 4. Cryptosystems 1 and 2 are homomorphic with respect to quasi-group operation $*$.

Proof. We shall use notations from Theorem 3.

Let's have a look at the element $W=\operatorname{Dec}\left(c_{1}\right) * \operatorname{Dec}\left(c_{2}\right)$. Using the definition of the operation Dec, we get:

$$
W=\left(s_{1}-{ }^{\left[a_{1}\right] \ldots\left[a_{r}\right]} g_{1}^{\left[a_{r+1}\right] \ldots\left[a_{n}\right]}\right) *\left(s_{2}-{ }^{\left[a_{1}\right] \ldots\left[a_{r}\right]} g_{2}^{\left[a_{r+1}\right] \ldots\left[a_{n}\right]}\right) .
$$

Let's apply the second statement of the Lemma 2:

$$
W=\left(s_{1} * s_{2}\right)-\left({ }^{\left[a_{1}\right] \ldots\left[a_{r}\right]} g_{1}^{\left[a_{r+1}\right] \ldots\left[a_{n}\right]} *\left[a_{1}\right] \ldots\left[a_{r}\right] g_{2}^{\left[a_{r+1}\right] \ldots\left[a_{n}\right]}\right) .
$$


If we apply the first statement from lemma 2 several times, we get:

$$
W=\left(s_{1} * s_{2}\right)-\left({ }^{\left[a_{1}\right] \ldots\left[a_{r}\right]}\left(g_{1} * g_{2}\right)^{\left[a_{r+1}\right] \ldots\left[a_{n}\right]}\right) .
$$

We see that $W$ coincides with the result of the decryption of the message $c_{1} * c_{2}=\left(g_{1} * g_{2}, s_{1} * s_{2}\right)$. Thus, $\operatorname{Dec}\left(c_{1} * c_{2}\right)=\operatorname{Dec}\left(c_{1}\right) * \operatorname{Dec}\left(c_{2}\right)$.

One can show that the built-up systems are not fully homomorphic. Yet they are homomorphic for many classes of functions.

For example, if we take the cyclic group $\left(\mathbb{Z}_{p},+\right)(p>2)$, the identical automorphism $\sigma$, and $\tau(x)=-x$, then we can prove that the proposed cryptosystems are homomorphic for an $n$-ary operation $*\left(a_{1}, \ldots, a_{n}\right)$ if and only if the operation $*$ is given by a linear function of $a_{1}, \ldots, a_{n}$ from $\left(\mathbb{Z}_{p},+\right)$.

\section{References}

[1] Belyavskaya G. B., Tabarov A. Kh., "Identities with permutations leading to linearity of quasigroups", Discrete Math. Appl., 19:2 (2009), 173-190.

[2] Babenko L. K., Byrtika F.B., Makarevitch O.B., Trapacheva A.V., "A fully homomorphic encoding (review)", Problems of information security, 3 (2016), 3-25, in Russian.

[3] Baryshnikov A.V., Katyshev S.Yu., "Key agreement schemes based on linear groupoids", Matematicheskie Voprosy Kriptografii, 8:1 (2017), 7-12.

[4] Baryshnikov A.V., Katyshev S.Yu., "Application of non-associative structures for the construction of public key distribution algorithms", Matematicheskie Voprosy Kriptografii, 9:4 (2018), 5-30, in Russian.

[5] Boneh D., Goh E.-J., Nissim K., "Evaluating 2-DNF formulas on ciphertexts", TCC'05, Lect. Notes Comput. Sci., 3378, 2005, 325-341.

[6] Gentry C., A Fully Homomorphic Encryption Scheme, Ph.D. Thesis. - Stanford Univ., 2009.

[7] Goldwasser S., Micali S., "Probabilistic encryption", J. Comput. System Sci., 28:2 (1984), 270-299.

[8] Gribov A.V., "Some homomorphic cryptosystem based on nonassociative structures", J. Math. Sci., 223:5 (2017), 581-586.

[9] El-Gamal T., "A public-key cryptosystem and a signature scheme based on discrete logarithms", IEEE Trans. Inform. Theory, 4 (1985), 469-472.

[10] Katyshev S. Yu., Markov V. T., Nechaev A. A, "Application of non-associative groupoids to the realization of an open key distribution procedure", Discrete Math. Appl., 25:1 (2015), $9-24$.

[11] Katyshev S. Yu., "Discrete logarithm problem in finite dimensional algebras over field", Prikladnaya diskretnaya matematika, 26:4 (2014), 21-28, in Russian.

[12] Rivest R., Adleman L., Dertouzos M., "On data banks and privacy homomorphisms", Foundations of Secure Computation. Academic Press, 1978, 169-177.

[13] Varnovsky N. P., Shokurov A.V., "Homomorphic Encryption", Trudy ISP RAN, 12 (2006), 27-36 (in Russian). 
